\title{
Knee Joint Moments Can Accurately Predict Medial and Lateral Knee Contact Forces in Patients With Valgus Malalignment
}

\author{
Jana Holder ( $\nabla$ jana.holder@kgu.de ) \\ University Hospital Frankfurt, Goethe University Frankfurt \\ Stefan Drongelen \\ University Hospital Frankfurt, Goethe University Frankfurt \\ Scott David Uhlrich \\ Stanford University \\ Eva Herrmann \\ Goethe University Frankfurt

\section{Andrea Meurer} \\ University Hospital Frankfurt, Goethe University Frankfurt \\ Felix Stief \\ University Hospital Frankfurt, Goethe University Frankfurt
}

\section{Research Article}

Keywords: Children and adolescents, valgus malalignment, musculoskeletal modeling, gait analysis, linear mixed-effects model

Posted Date: February 21st, 2022

DOl: https://doi.org/10.21203/rs.3.rs-1366268/v1

License: (9) (1) This work is licensed under a Creative Commons Attribution 4.0 International License. Read Full License 


\section{Abstract}

Compressive knee joint contact force during walking is thought to be related to initiation and progression of knee osteoarthritis. However, joint loading is often evaluated with surrogate measures, like the external knee adduction moment, due to the complexity of computing joint contact forces. Statistical models have shown promise for predicting joint contact force from easily measured joint moments in individuals with osteoarthritis or joint replacements. This approach may also be effective in young patients with valgus deformities. The purpose of this study was to evaluate how accurately medial and lateral knee joint contact forces could be estimated by linear mixed-effects models during walking for children with and without valgus malalignment. Knee joint moments were strongly correlated $\left(R^{2}>0.85, p<0.001\right)$ with both medial and lateral knee joint contact forces. The knee flexion and adduction moments were significant covariates in most of the models, strengthening the understanding of the contribution of both moments to medial and lateral knee joint contact force. In the future, these models could be used to predict knee joint contact forces using joint moments from motion capture software, obviating the need for time-consuming musculoskeletal simulations.

\section{Introduction}

In the last five years the number of studies has tripled (see supplementary material for the full search terms used in Pubmed) that performed sports or clinical gait analysis and investigated internal joint contact or muscle forces rather than joint moments ${ }^{1}$. Both joint moments and joint contact forces aim to characterize cartilage loading during dynamic movements ${ }^{2}$. The knee adduction moment (KAM) is a commonly used surrogate measure for medial compartment knee loading because it is related to osteoarthritis $(\mathrm{OA})$ initiation and progression ${ }^{3,4}$ and is relatively simple to compute. Reducing the external KAM is often the target for nonsurgical interventions, like changing the foot progression angle or by increasing the step width ${ }^{5}$, that aim to reduce medial compartment loading. However, the external KAM only characterizes the effects of external forces (e.g., ground reaction force), frontal-plane kinematics, and segment inertia on the distribution of loading between the medial and lateral compartments ${ }^{6}$. In contrast, internal joint forces, like the medial and lateral knee joint contact force (medKCF, latKCF), additionally represent the forces that oppose muscle forces ${ }^{7,8}$.

Both methods for estimating joint loading demonstrate advantages: joint moments are easily calculated, but knee contact forces are more representative of cartilage loading. Joint moments are usually available almost directly after the movement analysis because this method is often implemented in standard movement analysis pipelines, which may explain its acceptance as a surrogate measure for the dynamic internal knee joint loading. The external knee flexion/extension moment (KFM/KEM) also contributes to the internal knee joint contact force (KCF) as it relates to knee flexor and extensor muscle force. Linear models that use both the KAM and KFM as covariates have higher correlations with KCF than models that use KAM alone. ${ }^{9-11}$ Calculating the internal joint contact, muscle and/or tendon forces requires additional time and expertise ${ }^{1,12,13}$. Therefore, the calculation of joint moments has the advantage of quick availability and lower cost in terms of time or human capacity. Estimating internal joint contact forces with musculoskeletal models includes the contribution of internal forces, like muscles and ligaments, making internal joint contact forces a potentially more accurate surrogate measure for dynamic joint loading. Nevertheless, both methods are estimations of the loading in a joint ${ }^{2}$. In vivo measurement of joint contact force can only be done by invasive methods as an instrumented prosthesis. Patients with instrumented prostheses are rare ${ }^{14,15}$, and their loading patterns may not be representative of other populations of interest, like children. Additionally, highly dynamic movements like side-cutting have not been investigated in patients with instrumented prosthesis. The other two named methods, calculating external joint moments and internal joint contact forces, are therefore used in a more dynamic environment when younger study cohorts and other dynamic movements except for walking are investigated.

In a clinical setting, methods for estimating joint loading that are both accurate and inexpensive are needed. Previous studies showed that with a valgus malalignment, the external KAM amd medKCF during walking are reduced ${ }^{16,17}$ whereas latKCF is increased ${ }^{18}$. Similarly, results were found in patients with medial knee OA who walk with an increased KAM ${ }^{19}$ and a larger medKCF compared to age-matched healthy controls ${ }^{20}$. The KAM and medKCF are highly correlated during first half of stance, but weaker

correlations exist between KAM and latKCF ${ }^{20-22}$. In general, the relationship between knee joint moments (KJMs) and latKCF has been less studied, and most cohorts are older adults or individuals with knee OA. ${ }^{23-25}$ The ability of joint moments to predict 
medKCF and latKCF in these cohorts is promising, but further work is needed to understand these relationships in young individuals with valgus malalignment who are at increased risk of developing $O A$.

The aim of this study was to develop statistical models that relate external knee joint moments, (i.e., the knee adduction and flexion moments) to internal knee joint contact forces (i.e., medial and lateral knee joint contact forces) during walking in young patients with and without valgus malalignment. We hypothesized that 1) the prediction accuracy of the knee contact forces for children with and without valgus malalignment from external joint moments is high $\left.{ }^{21,26}\left(R^{2}>0.49 ; \mathrm{RMSE}<10 \%\right) ; 2\right)$ the prediction accuracy for the medial knee joint contact force by the external knee joint moments is larger compared to the lateral knee joint contact force; and 3) the prediction accuracy of the statistical models that use both sagittal and frontal plane moments to predict the knee joint contact forces will be greater than those that use joint moments from a single plane.

\section{Results}

\section{Anthropometrics and walking speed}

For comparing the anthropometrics and walking speed between groups, we performed independent $t$-tests or Mann-Whitney- $U$-test for not normally distributed data. These results are summarized in Table 1. All parameters except for age were normally distributed. The study groups were significant different in body height $(p=0.014)$, body mass $(p<0.001)$, body mass index ( $p<$ $0.001)$ and the mechanical axis angle $(p<0.001)$ but not for age and walking speed $(p>0.05)$. The effect sizes were large for all parameters except for age, body height and walking speed.

Table 1

Anthropometrics and walking speed.

\begin{tabular}{|c|c|c|c|c|c|c|}
\hline & \multicolumn{2}{|l|}{ Patient group } & \multicolumn{2}{|l|}{ TD group } & \multicolumn{2}{|c|}{ Comparison between groups } \\
\hline & & $\begin{array}{l}\text { Shapiro- } \\
\text { Wilk }\end{array}$ & & $\begin{array}{l}\text { Shapiro- } \\
\text { Wilk }\end{array}$ & $\begin{array}{l}t \text {-test / Mann-Whitney- } \\
\text { U-test }\end{array}$ & $\begin{array}{l}\text { Effect } \\
\text { size } r\end{array}$ \\
\hline & & (p-value) & & ( $p$-value) & (p-value) & \\
\hline Number of participants & 50 & & 21 & & & \\
\hline Sex [female / male] & 19 / 31 & & $7 / 14$ & & & \\
\hline $\begin{array}{l}\text { Bilateral / left / right affected } \\
\text { limbs }\end{array}$ & $38 / 5 / 7$ & & $-/ 10 / 11$ & & & \\
\hline \multirow[t]{2}{*}{ Age [years] } & 13.0 & $<0.001$ & 12.0 & 0.004 & 0.294 * & 0.124 \\
\hline & $\begin{array}{l}(11.0- \\
13.0)\end{array}$ & & $\begin{array}{l}(12.0- \\
14.0)\end{array}$ & & & \\
\hline Body height [m] & $1.66 \pm 0.10$ & 0.379 & $1.59 \pm 0.10$ & 0.612 & 0.014 & 0.291 \\
\hline Body mass $[\mathrm{kg}]$ & $63.5 \pm 13.7$ & 0.680 & $46.1 \pm 10.7$ & 0.364 & $<0.001$ & 0.530 \\
\hline Body mass index $\left[\mathrm{kg} / \mathrm{m}^{2}\right]$ & $23.0 \pm 3.4$ & 0.204 & $18.1 \pm 2.5$ & 0.132 & $<0.001$ & 0.585 \\
\hline Mechanical axis angle $\left[^{\circ}\right]$ & $-6.0 \pm 1.8$ & 0.248 & $-0.0 \pm 2.3$ & 0.379 & $<0.001$ & 0.811 \\
\hline Walking speed [m/s] & $1.25 \pm 0.16$ & 0.569 & $1.29 \pm 0.17$ & 0.810 & 0.368 & 0.109 \\
\hline
\end{tabular}

TD: Typically developed healthy control group; Mechanical axis angle of the patients was measured by an X-ray image; mechanical axis angle of the TD group was measured from the static trial from the three-dimensional gait analysis; Normal distributed data displayed as mean \pm standard deviation; not normally distributed data are displayed as median (25. quartile - 75 . quartile) and marked with a *; Mann-Whitney-Utests have been performed instead of independent $t$-tests for not normally distributed data; significant $p$-values are highlighted in bold; Effect size $r>0.1$ : small; $r>0.3$ : medium; $r>0.5$ : strong. 
The mean curves of the dynamic KJMs and KCFs were compared between the two groups using statistical parametric mapping (Fig. 1). The KFM did not significantly differ between the patient and typically developed healthy control (TD) group. KAM was significantly smaller in the patient group between $3-52 \%(p<0.001)$ and $61-66 \%(p=0.010)$ of the gait cycle. The medKCF was significantly smaller in the patient group between $0-25 \%(p<0.001)$, between $46-52 \%(p=0.005)$, between $58-88 \%(p<0.001)$, and between $91-100 \%(p<0.001)$. The latKCF was significantly increased for the patient group between $37-50 \%(p<0.001)$ and between $75-80 \%(p=0.018)$ of the gait cycle. Other kinematic and kinetic curves and comparisons are included in the supplementary material.

\section{Linear models}

To establish the relationships between single-plane KJMs and KCFs, we first investigated correlations of KAM or KFM and medKCF or latKCF for the peaks in the first and second half of stance individually. Low to moderate correlations of $R^{2}<0.49$ were detected except between KAM2 and latKCF2 $\left(R^{2}=0.68\right)$ for the patient group and KAM2 and medKCF2 $\left(R^{2}=0.59\right)$ for the TD group. The root mean squared error (RMSE) ranged between 14-29\%. See full results in the supplementary material, Table 1 and Table 2.

\section{Linear mixed-effects models}

For testing the possibility of accurately predicting peaks of medKCF and latKCF by combining KAM and KFM/KEM, we used linear mixed-effects models (LMM). For improvement of the model, random effects for both included limbs from bilaterally affected patients and different numbers of included trials per participant were added.

\section{Patients}

Equations 1 to 4 describe the LMMs that relate knee moments to the first and second peaks of medKCF and latKCF in the patient group. The first and second peaks of the variables are denoted by appending the peak number to the end of the variable (e.g., the first peak KAM is KAM1). The results of the LMMs are summarized in Table 2. For all four LMMs, KAM, and the squared knee flexion/extension moment (qKFM1, qKEM2) were included as significant fixed and random effects. The first model describing the relationship between medKCF1 and KAM1 and qKFM1 reported an adjusted $R^{2}=0.90$ (Eq. 1). The relationship between KAM1 and qKFM1 and the first peak of latKCF was also strong with an adjusted $R^{2}=0.89$ (Eq. 3). For the second peak of medKCF and latKCF, both LMMs were highly correlated with an adjusted $R^{2}=0.96$ (Eq. 2) and $R^{2}=0.95$ (Eq. 4), respectively.

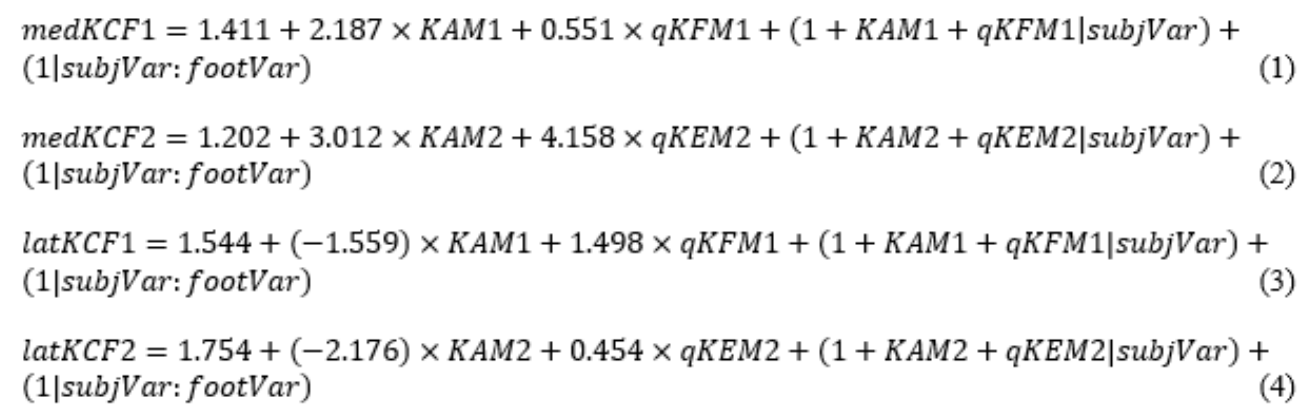


Table 2

Statistical summary of the linear mixed-effects models between the internal knee joint contact forces and external knee joint moments for the patient group.

\begin{tabular}{|c|c|c|c|c|c|c|c|c|c|c|c|}
\hline $\begin{array}{l}\text { Response } \\
\text { Variable }\end{array}$ & $\begin{array}{l}\text { Predictor } \\
\text { Variable }\end{array}$ & Estimate & $\begin{array}{l}\text { Standard } \\
\text { Error }\end{array}$ & $t$-value & $\begin{array}{l}\text { Degrees } \\
\text { of } \\
\text { freedom }\end{array}$ & $p$-value & $\begin{array}{l}\text { Lower } \\
95 \% \\
\mathrm{Cl}\end{array}$ & $\begin{array}{l}\text { Upper } \\
95 \% \\
\text { Cl }\end{array}$ & $\begin{array}{l}\text { Adj. } \\
R^{2}\end{array}$ & RMSE & $\begin{array}{l}\text { RMSE } \\
\text { [\%] }\end{array}$ \\
\hline \multirow[t]{3}{*}{ medKCF1 } & Intercept & 1.411 & 0.049 & 29.009 & 417 & $<0.001$ & 1.315 & 1.506 & 0.90 & 0.14 & 7.01 \\
\hline & KAM1 & 2.187 & 0.166 & 13.206 & 417 & $<0.001$ & 1.861 & 2.513 & & & \\
\hline & qKFM1 & 0.551 & 0.073 & 7.513 & 417 & $<0.001$ & 0.407 & 0.695 & & & \\
\hline \multirow[t]{3}{*}{ medKCF2 } & Intercept & 1.202 & 0.054 & 22.272 & 417 & $<0.001$ & 1.096 & 1.308 & 0.96 & 0.13 & 6.0 \\
\hline & KAM2 & 3.012 & 0.228 & 13.184 & 417 & $<0.001$ & 2.563 & 3.462 & & & \\
\hline & qKEM2 & 4.158 & 0.209 & 19.863 & 417 & $<0.001$ & 3.747 & 4.570 & & & \\
\hline \multirow[t]{3}{*}{ latKCF1 } & Intercept & 1.544 & 0.044 & 35.012 & 417 & $<0.001$ & 1.457 & 1.630 & 0.89 & 0.15 & 10.3 \\
\hline & KAM1 & -1.559 & 0.162 & -9.649 & 417 & $<0.001$ & -1.876 & -1.241 & & & \\
\hline & qKFM1 & 1.498 & 0.117 & 12.819 & 417 & $<0.001$ & 1.269 & 1.728 & & & \\
\hline \multirow[t]{3}{*}{ latKCF2 } & Intercept & 1.754 & 0.042 & 41.818 & 417 & $<0.001$ & 1.671 & 1.836 & 0.95 & 0.10 & 6.8 \\
\hline & KAM2 & -2.176 & 0.135 & -16.146 & 417 & $<0.001$ & -2.441 & -1.911 & & & \\
\hline & qKEM2 & 0.454 & 0.141 & 3.218 & 417 & 0.001 & 0.177 & 0.731 & & & \\
\hline
\end{tabular}

Cl: Confidence interval; Adj. $R^{2}$ a adjusted $R^{2} ; R M S E$ : root mean squared error [N/(kg×ms $\left.\left.{ }^{-2}\right)\right]$; medKCF1/medKCF2: max. value in the first/second half of stance of the medial knee joint contact force [N/(kg×ms $\left.\left.{ }^{-2}\right)\right]$; latKCF1/latKCF2: max. value in the first/second

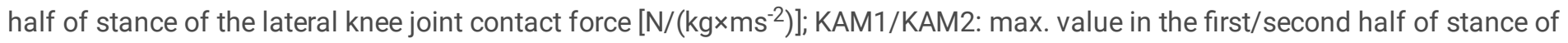
the external knee adduction moment [Nm/kg]; qKFM1/qKEM2: squared maximal/minimal value in the first/second half of stance of the external knee flexion/extension moment (KFM1, KEM2; [Nm/kg]).

\section{Typically developed healthy controls}

For the TD group LMMs were also performed for the four parameters medKCF1, medKCF2, latKCF1 and latKCF2 and shown in Eq. 5 to 8. Similar $R^{2}$ values were found with the chosen model (medKCF1: $R^{2}=0.95$ (Eq. 5); medKCF2: $R^{2}=0.97$ (Eq. 6); latKCF1: $R^{2}=$ 0.93 (Eq. 7); latKCF2: $\mathrm{R}^{2}=0.92$ (Eq. 8)) compared to the results from the patient group (Table 3).

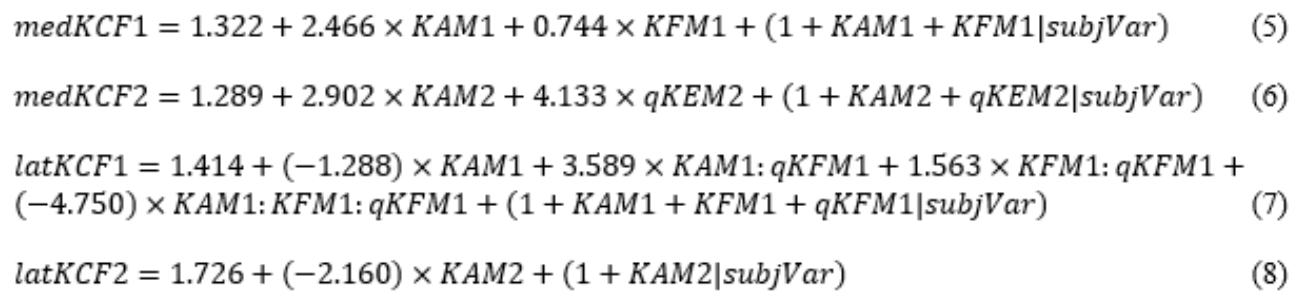


Table 3

Statistical summary of the linear mixed-effects models between the internal knee joint contact forces and external knee joint moments for the typically developed healthy control group.

\begin{tabular}{|c|c|c|c|c|c|c|c|c|c|c|c|}
\hline Response & Predictor & Estimate & Standard & $t$-value & Degrees & $p$-value & Lower & Upper & Adj. & RMSE & RMSE \\
\hline Variable & Variables & & Error & & $\begin{array}{l}\text { of } \\
\text { Freedom }\end{array}$ & & $\begin{array}{l}95 \% \\
\mathrm{Cl}\end{array}$ & $\begin{array}{l}95 \% \\
\mathrm{Cl}\end{array}$ & $R^{2}$ & & [\%] \\
\hline \multirow[t]{3}{*}{ medKCF1 } & Intercept & 1.322 & 0.142 & 9.307 & 93 & $<0.001$ & 1.040 & 1.604 & 0.95 & 0.13 & 5.1 \\
\hline & KAM1 & 2.466 & 0.413 & 5.965 & 93 & $<0.001$ & 1.645 & 3.287 & & & \\
\hline & KFM1 & 0.744 & 0.128 & 5.822 & 93 & $<0.001$ & 0.490 & 0.997 & & & \\
\hline \multirow[t]{3}{*}{ medKCF2 } & Intercept & 1.289 & 0.123 & 10.495 & 93 & $<0.001$ & 1.045 & 1.533 & 0.97 & 0.13 & 4.7 \\
\hline & KAM2 & 2.902 & 0.485 & 5.984 & 93 & $<0.001$ & 1.939 & 3.865 & & & \\
\hline & qKEM2 & 4.133 & 0.483 & 8.550 & 93 & $<0.001$ & 3.173 & 5.093 & & & \\
\hline \multirow[t]{9}{*}{ latKCF1 } & Intercept & 1.414 & 0.166 & 8.515 & 91 & $<0.001$ & 1.084 & 1.743 & 0.93 & 0.11 & 9.3 \\
\hline & KAM1 & -1.288 & 0.500 & -2.576 & 91 & 0.012 & -2.281 & -0.295 & & & \\
\hline & KAM1: & 3.589 & 1.431 & 2.508 & 91 & 0.014 & 0.746 & 6.431 & & & \\
\hline & qKFM1 & & & & & & & & & & \\
\hline & KFM1: & 1.563 & 0.510 & 3.067 & 91 & 0.003 & 0.551 & 2.575 & & & \\
\hline & qKFM1 & & & & & & & & & & \\
\hline & KAM1: & -4.750 & 1.242 & -3.823 & 91 & $<0.001$ & -7.218 & -2.282 & & & \\
\hline & KFM1: & & & & & & & & & & \\
\hline & qKFM1 & & & & & & & & & & \\
\hline \multirow[t]{2}{*}{ latKCF2 } & Intercept & 1.726 & 0.094 & 18.372 & 94 & $<0.001$ & 1.539 & 1.912 & 0.92 & 0.11 & 9.5 \\
\hline & KAM2 & -2.160 & 0.279 & -7.745 & 94 & $<0.001$ & -2.714 & -1.607 & & & \\
\hline
\end{tabular}

Cl: Confidence interval; Adj. $R^{2}$ : adjusted $R^{2} ; R M S E$ : root mean squared error [N/(kg×ms $\left.\left.{ }^{-2}\right)\right]$; medKCF1/medKCF2: max. value in the

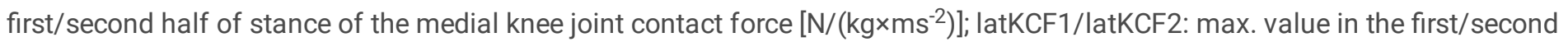
half of stance of the lateral knee joint contact force $\left[\mathrm{N} /\left(\mathrm{kg} \times \mathrm{ms}^{-2}\right)\right]$; KAM1/KAM2: max. value in the first/second half of stance of the external knee adduction moment [Nm/kg]; qKFM1/qKEM2: squared maximal/minimal value in the first/second half of stance of the external knee flexion/extension moment (KFM1, KEM2; [Nm/kg]).

Similar trends of LMM structures between the patient and TD group were observed. The knee adduction moment plays a major role for predicting medKCF1 and latKCF2. Predicting medKCF2, KEM2 was found to have a larger impact compared to KAM2. Only for latKCF1 different structures between the patient and TD group were seen. The lateral KCF was negatively correlated with KAM for both peaks in patients and TDs.

\section{Discussion}

We investigated the accuracy of statistical models that predict internal knee joint contact forces from knee joint moments in children and adolescents with and without valgus malalignment. We found that the models could predict medial and lateral knee contact force peaks with a high accuracy of $R^{2}>0.85$ and $R M S E<11 \%$ when including knee joint moments from both the sagittal and frontal plane. The first hypothesis was confirmed that for both study groups, the knee contact forces can be predicted with high accuracy with their individual statistical models. The second hypothesis was rejected, because both medial and lateral knee contact force, could be predicted with high accuracy $\left(R^{2}>0.85\right)$ from joint moments. Our third hypothesis was confirmed, as 
models that included knee moments in the sagittal and frontal plane predicted joint contact forces with higher accuracy $\left(R^{2}=\right.$ $0.89-0.97)$ than those that used a moment in a single plane $\left(R^{2}=0.01-0.68\right)$. These results suggest that internal knee joint loading can be accurately predicted with statistical models that use inputs from commonly-used gait analysis tools, mitigating the need for complex musculoskeletal modeling procedures, potentially enabling these estimates to be made in a clinical setting.

Previous studies found that KAM correlated well with medKCF in the first half of stance with a prediction accuracy of about $R^{2} \approx$ 0.4 and were performed in patients with knee $\mathrm{OA}$ or after knee replacement ${ }^{22,24,27}$. Previous studies also investigated the relationship between KFM and medKCF with, in general, low correlations $\left(R^{2}<0.25\right)$ for both peaks ${ }^{20,24,28,29}$. Moreover, in few studies, multivariate models were used to study the effect of KAM and KFM on medKCF. These studies improved the prediction of medKCF for the first peak ( $R^{2}$ improved by approximately 0.2 ) but not for the second peak. In general, the reported $R^{2}$ for the first peak of medKCF using KAM and KFM varied between 0.54 and 0.85 in older adults with and without musculoskeletal pathologies ${ }^{10,11,21}$. The lateral knee joint contact force has been less investigated and the few studies performed only found low relations with $\mathrm{KAM}\left(R^{2}<0.15\right)$ and slightly stronger correlations with $\mathrm{KFM}\left(R^{2}<0.3\right)^{23-25}$. In the present study, for both study groups the LMMs revealed large predictive power of $R^{2}>0.88$ and a RMSE $\leq 10.3 \%$. These results strengthen the possibility of an accurate determination of internal KCFs by external KAM and KFM/KEM for young individuals with and without valgus malalignment.

Studies found that reducing KAM with gait modifications does not necessarily also change medKCF because other joint loading parameters as KFM or muscle co-contraction might be affected ${ }^{11,30,31}$. A possible successful gait modification for reducing medKCF could be in-toeing that potentially reduces KAM but not substantially affecting KFM ${ }^{32-34}$. The effect of gait modifications on latKCF has not been investigated in the past. Previous studies found KFM or KEM as the main contributor to latKCF ${ }^{35}$, suggesting that offloading gait should target these parameters. ${ }^{31,33}$ All four statistical models from the present study revealed a negatively directed correlation between KAM and latKCF. This suggests that an increase of KAM and no change of KFM could reduce latKCF. Nonetheless, gait modifications often alter both KAM and KFM and affect both medKCF and latKCF; though, the relative contribution of the moments differ between compartments and peak times. Future studies should use models, like the ones presented here, that consider the effects of both KAM and KFM on KCF.

Most other studies relating KJMs to KCFs investigate older populations with knee OA who likely have varus or neutral frontal plane alignment ${ }^{27}$. Our results provide estimates of loading in young patients with valgus malalignment that may inform the need for guided growth intervention in these children. Currently, the decision for a guided growth intervention is based on the static mechanical axis angle from an X-ray image, which is not highly correlated with medKCF and latKCF ${ }^{18}$. Consequently, this study may be helpful in the decision-making for guided growth. Moreover, the coefficients of our models are mostly different from those in models that used patients with varus alignment. In our models, the extracted values of KFM/KEM were included as squared parameters because a high variance was found. This highlights the importance of using population-specific models to determine internal KCFs. ${ }^{20,36,37}$

\section{Limitations}

It is important to identify the limitations of the study. With the linear regression model, we aimed to predict the KCFs by only using the KJMs. This study demonstrates that KCFs can be accurately predicted using KJMs from OpenSim; however, a more clinically applicable solution would be to use the KJMs directly from the three-dimensional motion capture system. We used KJMs from OpenSim to avoid confounding effects of differing coordinate systems, which can influence kinematic and kinetic results ${ }^{38,39}$. Future studies should investigate the influence of different coordinate systems or models on the relationship between joint moments and joint contact forces. Alternatively, a transformation between the motion capture and OpenSim coordinate systems could be determined and applied to the joint moment data prior to using our model. Moreover, to be able to include further parameters in this linear regression analysis, the dataset needs to be increased, especially for the TD group. Lastly, for fulfilling a complete view of leg alignment in children and adolescents and the influence of leg alignment on internal joint contact force, patients with varus alignment should be included. Although, in our hospital children and adolescents with varus alignment are also part of a large study, the number of participants is still small and therefore were not included. 


\section{Conclusion}

We investigated the relationship between knee joint moments and knee joint contact forces in children with and without valgus malalignment during walking. The predictions from linear models were strongly related to knee joint contact forces from musculoskeletal simulations. This suggests that knee joint contact forces could be estimated in the future using knee joint moments from standard motion capture software as input to the linear models. Furthermore, including both the knee flexion/extension and adduction moments in the linear models increased the prediction accuracy. This supports the importance of evaluating the role of both muscle forces and dynamic mechanics in medial and lateral knee joint contact forces. By simplifying the evaluation of internal joint loading, the statistical models may enable clinicians and researchers to study and prescribe gait modifications that reduce knee joint contact force without needing to perform time-consuming musculoskeletal simulations.

\section{Methods And Materials}

\section{Participants}

In total, 71 children and adolescents were included in this study, 50 of them with a valgus malalignment of the knee joint and 21 TDs (Table 1). Solely patients with a clinical indication for a temporary hemiepiphysiodesis were included. More specifically, a pathological valgus alignment of at least one knee (38 patients were bilaterally affected) of the lower limb based on a full-length standing anteroposterior radiograph was necessary $17,40,41$. In our hospital, the indication for a temporary hemiepiphysiodesis is given when the deviation from the physiological mechanical bearing line was more than $10 \mathrm{~mm}^{42}$, which is approximately $3^{\circ}$ deviation of the physiological mechanical axis angle. The static mechanical axis angle was measured as the angle formed by the line from the hip center to the knee center and the line from the knee center to the ankle center ${ }^{40}$. Patients did not show any other pathological disorders at the lower limb as described previously ${ }^{18}$. The participants for the TD study cohort were recruited from local schools. All participants and their parents were thoroughly familiarized with the gait analysis protocol. Participants and their parents gave written informed consent to participate in this study, as approved by the local ethics committee of the Goethe University Frankfurt, Germany (182/16) and in accordance with the Helsinki Declaration. The study is registered in the German Clinical Trials Register (DRKS) (number: DRKS00010296).

\section{Gait analysis}

Kinematic data were collected barefoot at $200 \mathrm{~Hz}$ using an 8-camera three-dimensional motion capture system (MX 10, VICON Motion Systems, Oxford, UK). Ground reaction forces were recorded synchronously at $1000 \mathrm{~Hz}$ using two force plates (Advanced Mechanical Technology, Inc., Watertown, MA, USA) situated at the mid-point of the $15 \mathrm{~m}$ long level walkway. When analyzing frontal and transverse plane gait data, a custom made lower body protocol was used for improvement of the reliability and accuracy described in a previous study ${ }^{43}$. In addition to the standardized Plug-in-Gait marker set ${ }^{44}$, reflective markers were attached on the medial malleolus, medial femoral condyle and greater trochanter. The statically measured midpoints between the medial and lateral malleolus and condyle markers defined the centers of rotation of the ankle and knee joints ${ }^{43}$. The center of the hip joint was calculated with a standardized geometrical prediction method using regression equations ${ }^{6}$ which is common in the clinical gait community ${ }^{45}$. During the static upright standing trial, participants stood barefoot, feet shoulder width apart, knees fully extended, in a forward knee position with the patella centered over the femoral condyles to control for any foot rotation effects ${ }^{46}$. Three to five dynamic trials with a clear foot-force plate contact were selected for further processing.

\section{Musculoskeletal modeling}

OpenSim (4.1) was used for musculoskeletal modeling of joint angles, joint moments, muscle activations, and forces and joint contact forces ${ }^{47}$. Input from marker positions and ground reaction forces were prepared with the MOtoNMS toolbox (version 3 ) in MATLAB (2020b, The MathWorks, Inc., Natick, MA, USA) for usage in OpenSim ${ }^{48}$. Ankle and knee joint centers were calculated in MOtoNMS. The joint centers were the midpoints between the medial and lateral malleolus and femoral condyle markers. Force data were filtered with a zero-lag low-pass Butterworth filter and a cut-off frequency of $10 \mathrm{~Hz}$. An OpenSim model ${ }^{7,49}$ with 23 degrees of freedom was used: six degrees of freedom for the pelvis relative to the ground frame, three for the lumbosacral joint, three for the hip joint, two for the knee joint, one for the ankle joint and one for the subtalar joint. The knee joint had sagittal and 
frontal-plane rotational degrees of freedom, and medial and lateral contact forces were resolved using a multi-compartment knee mode ${ }^{8,50}$. The model was actuated by 80 muscle-tendon actuators ${ }^{7,51}$ and passive muscle force-length curves were calibrated using experimental data ${ }^{49,52}$.

The generic musculoskeletal model was linearly scaled based on marker positions and participant anthropometrics. Models were further personalized by adjusting the neutral frontal-plane alignment with the mechanical axis angle measured from X-Ray images $^{8}$. X-rays were not available for the TD group, and the mechanical axis angle was calculated using a static gait analysis trial. ${ }^{18,53}$. It has been shown that this non-invasive marker-based approach correlated well with the determination of lower limb alignment in the frontal plane using radiographs in young patients with varus or valgus malalignment ${ }^{53}$. Inverse kinematics and inverse dynamics were calculated with the standard OpenSim processing pipelines. A static optimization implementation that incorporates tendon compliance and passive muscle forces was used to solve for muscle activations, with a cost function that minimized the sum of squared muscle activation ${ }^{49}$. Knee contact forces were computed and are reported as the reaction force in the medial and lateral compartments of the knee in the direction of the long axis of the tibia. All calculations were performed in MATLAB. Kinematic and kinetic parameters were segmented by gait cycle. External KJMs were normalized by body mass and KCFs were normalized by bodyweight.

\section{Statistical analysis}

The anthropometrics (age, body height, body mass, body mass index and the mechanical axis angle) and the walking speed of the patient group and the TD group were tested for normality using a Shapiro-Wilk test. Differences between patients and TDs of normal distributed data were compared with an independent $t$-test and non-normal distributed data with a Mann-Whitney-U-test (SPSS, 26, IBM Corporation, New York, NY, USA). The effect size $r$ of the anthropometrics and the walking speed was calculated ${ }^{54}$. $r>0.1$ described a small effect size, $r>0.3$ a medium effect size and $r>0.5$ a large effect size ${ }^{55}$. KJM and KCF mean curves between groups were statistically tested for normality and compared using a two-sample parametric $t$-test within statistical parametric mapping ${ }^{56}$ in MATLAB. Significant differences were considered when the critical threshold of $a=0.05$ was passed for more than four successive time points, i.e. at least $4 \%$ of the gait cycle ${ }^{57}$. In the patient group, 38 participants were bilaterally affected by valgus malalignment. For the mechanical axis angle, walking speed, kinematic and kinetic comparisons between the study groups, only the more affected limb in regards of the mechanical axis angle was included. For the LMMs, both affected limbs were included. For TD, only one leg was randomly chosen to be included in all performed analyses.

For investigating the linear relationship between external KJMs and KCFs, maximal values in the first and second half of stance of the medKCFs and latKCFs and KAMs were detected (medKCF1, medKCF2, etc.). For KJM in the sagittal plane the maximal value in the first half and the minimal value of the second half of stance were extracted (KFM1 and KEM2). First, linear regression analyses between one predictor (e.g. KAM) and the response (e.g. medKCF) variable for the peaks in the first and second half of stance were performed. The detailed description of this analysis is reported in the supplementary material (paragraph "Linear regression analysis"). Next, LMMs were used to include multiple predictor variables and to account for both included limbs in bilateral affected patients as well as different numbers of trials included per participant and limb. In general, a minimum of three and a maximum of five trials per leg were included in the analysis. The joint moments were included as fixed and random effects. Categorical variables for the participants (subjVar) and for the analyzed leg (footVar) were implemented as random effects associating each trial with an ascending participant number and the analyzed foot (left: 1, right: 2). The KFM peaks were additionally included as squared parameters qKFM1 or qKEM2 because extracted small KFM/KEM values showed deviations from a linear trend what was checked visually. medKCF1/medKCF2 and latKCF1/latKCF2 were selected as response variables. In total, four LMMs were built for both study cohorts. For finding the best fitted LMM for the four response variables, backward selection of all included parameters (KAM, KFM, qKFM) was performed. This means that the parameters KAM, KFM, qKFM and all their possible interactions were included in the first fitted LMM. Step by step, non-significant fixed effects have been removed from the model until only significant related effects have been left. Additionally, only fixed effects that significantly improved $R^{2}$ were included in the model to keep the models as small as possible. Random effects were excluded from the model when the variance was very small, as identified by visual inspection (approximately 10 times smaller than the variance of the residuals). In this study, the estimates, standard errors, $p$-values, the lower and upper bounds of the $95 \%$ confidence intervals, the adjusted $R^{2}$, the root

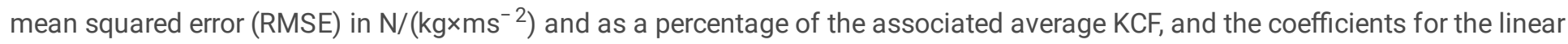


regression equation are reported for each LMM. Adjusted $R^{2} \leq 0.09$ were interpreted as little, $0.09<R^{2} \leq 0.25$ as low, $0.25<R^{2} \leq$

0.49 as moderate, $0.49<R^{2} \leq 0.81$ as high, and $R^{2}>0.81$ as very high correlations ${ }^{26}$. Statistical significance for all tests was set to $a=0.05$.

\section{List Of Abbreviations}

KAM knee adduction moment

KCF knee joint contact force

KFM/KEM knee flexion/extension moment

KJM knee joint moment

latKCF lateral knee joint contact force

LMM linear mixed-effects model

medKCF medial knee joint contact force

qKFM/qKEM squared knee flexion/extension moment

TD typically developed healthy controls

\section{Declarations}

\section{Data availability}

The datasets generated and analyzed during the current study are available from the corresponding author on reasonable request.

\section{Author contributions}

J. H., F. S and A. M. conceived the presented idea. J. H. and F. S. and S. v. D. performed the data collection, analysis and interpretation. J. H, S. v. D and S. D. U. performed the modeling procedures. J. H. performed the statistical analysis and F. S., S. v. D. and E. H. checked and approved it. J. H. drafted the manuscript and visualized the results. All authors reviewed the manuscript, suggested improvements in the content and approved the final version. All authors agreed to be accountable for all aspects of the work.

\section{Funding}

This work was funded by the Deutsche Forschungsgemeinschaft (DFG, German Research Foundation) - Project number: 403837822. Open Access funding enabled and organized by Project DEAL. Sponsor had no involvement in this article.

\section{Conflict of Interest}

The authors declare that the research was conducted in the absence of any commercial or financial relationships that could be construed as a potential conflict of interest.

\section{References}

1. Smith, S. H. L., Coppack, R. J., van den Bogert, A. J., Bennett, A. N. \& Bull, A. M. J. Review of musculoskeletal modelling in a clinical setting: Current use in rehabilitation design, surgical decision making and healthcare interventions. Clin Biomech (Bristol, Avon) 83, 105292, doi:10.1016/j.clinbiomech.2021.105292 (2021).

2. Baltzopoulos, V. Inverse dynamics, joint reaction forces and loading in the musculoskeletal system: guidelines for correct mechanical terms and recommendations for accurate reporting of results. Sports Biomech, 1-14, 
doi:10.1080/14763141.2020.1841826 (2021).

3. Andriacchi, T. P. \& Mündermann, A. The role of ambulatory mechanics in the initiation and progression of knee osteoarthritis. Curr Opin Rheumatol 18, 514-518, doi:10.1097/01.bor.0000240365.16842.4e (2006).

4. Miyazaki, T. et al. Dynamic load at baseline can predict radiographic disease progression in medial compartment knee osteoarthritis. Ann Rheum Dis 61, 617-622, doi:10.1136/ard.61.7.617 (2002).

5. Stief, F. et al. Impact of subject-specific step width modification on the knee and hip adduction moments during gait. Gait Posture 89, 161-168, doi:10.1016/j.gaitpost.2021.07.008 (2021).

6. Davis, R. B., Ounpuu, S., Tyburski, D. \& Gage, J. R. A Gait Analysis Data-Collection and Reduction Technique. Hum Mov Sci 10, 575-587, doi:Doi 10.1016/0167-9457(91)90046-Z (1991).

7. Rajagopal, A. et al. Full-Body Musculoskeletal Model for Muscle-Driven Simulation of Human Gait. IEEE Trans Biomed Eng 63, 2068-2079, doi:10.1109/TBME.2016.2586891 (2016).

8. Lerner, Z. F., DeMers, M. S., Delp, S. L. \& Browning, R. C. How tibiofemoral alignment and contact locations affect predictions of medial and lateral tibiofemoral contact forces. J Biomech 48, 644-650, doi:10.1016/j.jbiomech.2014.12.049 (2015).

9. Meireles, S. et al. Knee contact forces are not altered in early knee osteoarthritis. Gait Posture 45, 115-120, doi:10.1016/j.gaitpost.2016.01.016 (2016).

10. Manal, K., Gardinier, E., Buchanan, T. S. \& Snyder-Mackler, L. A more informed evaluation of medial compartment loading: the combined use of the knee adduction and flexor moments. Osteoarthritis Cartilage 23, 1107-1111, doi:10.1016/j.joca.2015.02.779 (2015).

11. Walter, J. P., D'Lima, D. D., Colwell, C. W., Jr. \& Fregly, B. J. Decreased knee adduction moment does not guarantee decreased medial contact force during gait. J Orthop Res 28, 1348-1354, doi:10.1002/jor.21142 (2010).

12. Kainz, H., Wesseling, M. \& Jonkers, I. Generic scaled versus subject-specific models for the calculation of musculoskeletal loading in cerebral palsy gait: Effect of personalized musculoskeletal geometry outweighs the effect of personalized neural control. Clin Biomech (Bristol, Avon) 87, 105402, doi:10.1016/j.clinbiomech.2021.105402 (2021).

13. Kainz, H. \& Schwartz, M. H. The importance of a consistent workflow to estimate muscle-tendon lengths based on joint angles from the conventional gait model. Gait Posture 88, 1-9, doi:10.1016/j.gaitpost.2021.04.039 (2021).

14. Kutzner, I. et al. Loading of the knee joint during activities of daily living measured in vivo in five subjects. J Biomech 43, 21642173, doi:10.1016/j.jbiomech.2010.03.046 (2010).

15. Kim, H. J. et al. Evaluation of predicted knee-joint muscle forces during gait using an instrumented knee implant. J Orthop Res 27, 1326-1331, doi:10.1002/jor.20876 (2009).

16. Stevens, P. M. Guided growth for angular correction: a preliminary series using a tension band plate. J Pediatr Orthop 27, 253259, doi:10.1097/BPO.0b013e31803433a1 (2007).

17. Farr, S., Kranzl, A., Pablik, E., Kaipel, M. \& Ganger, R. Functional and radiographic consideration of lower limb malalignment in children and adolescents with idiopathic genu valgum. J Orthop Res 32, 1362-1370, doi:10.1002/jor.22684 (2014).

18. Holder, J. et al. Effect of guided growth intervention on static leg alignment and dynamic knee contact forces during gait. Gait Posture 78, 80-88, doi:10.1016/j.gaitpost.2020.03.012 (2020).

19. Mündermann, A., Dyrby, C. O., Hurwitz, D. E., Sharma, L. \& Andriacchi, T. P. Potential strategies to reduce medial compartment loading in patients with knee osteoarthritis of varying severity: reduced walking speed. Arthritis Rheum 50, 1172-1178, doi:10.1002/art.20132 (2004).

20. Kumar, D., Manal, K. T. \& Rudolph, K. S. Knee joint loading during gait in healthy controls and individuals with knee osteoarthritis. Osteoarthritis Cartilage 21, 298-305, doi:10.1016/j.joca.2012.11.008 (2013).

21. Richards, R. E., Andersen, M. S., Harlaar, J. \& van den Noort, J. C. Relationship between knee joint contact forces and external knee joint moments in patients with medial knee osteoarthritis: effects of gait modifications. Osteoarthritis Cartilage 26, 12031214, doi:10.1016/j.joca.2018.04.011 (2018).

22. Holder, J., Trinler, U., Meurer, A. \& Stief, F. A Systematic Review of the Associations Between Inverse Dynamics and Musculoskeletal Modeling to Investigate Joint Loading in a Clinical Environment. Front Bioeng Biotechno/ 8, 603907, doi:10.3389/fbioe.2020.603907 (2020).

Page $11 / 14$ 
23. Noyes, F. R., Schipplein, O. D., Andriacchi, T. P., Saddemi, S. R. \& Weise, M. The anterior cruciate ligament-deficient knee with varus alignment. An analysis of gait adaptations and dynamic joint loadings. Am J Sports Med 20, 707-716, doi:10.1177/036354659202000612 (1992).

24. Winby, C. R., Gerus, P., Kirk, T. B. \& Lloyd, D. G. Correlation between EMG-based co-activation measures and medial and lateral compartment loads of the knee during gait. Clin Biomech (Bristol, Avon) 28, 1014-1019, doi:10.1016/j.clinbiomech.2013.09.006 (2013).

25. Saxby, D. J. et al. Tibiofemoral contact forces during walking, running and sidestepping. Gait Posture 49, 78-85, doi:10.1016/j.gaitpost.2016.06.014 (2016).

26. Hinkle, D. E., Jurs, S. G. \& Wiersma, W. Applied Statistics for the Behavioral Sciences. 2 edn, (Houghton Mifflin, 1988).

27. Kutzner, I., Trepczynski, A., Heller, M. O. \& Bergmann, G. Knee adduction moment and medial contact force-facts about their correlation during gait. PLoS One 8, e81036, doi:10.1371/journal.pone.0081036 (2013).

28. Wellsandt, E. et al. Predictors of knee joint loading after anterior cruciate ligament reconstruction. J Orthop Res 35, 651-656, doi:10.1002/jor.23408 (2017).

29. Ogaya, S. et al. Knee adduction moment and medial knee contact force during gait in older people. Gait Posture 40, 341-345, doi:10.1016/j.gaitpost.2014.04.205 (2014).

30. Lindsey, B., Eddo, O., Caswell, S. V., Prebble, M. \& Cortes, N. Reductions in peak knee abduction moment in three previously studied gait modification strategies. Knee 27, 102-110, doi:10.1016/j.knee.2019.09.017 (2020).

31. Booij, M. J., Richards, R., Harlaar, J. \& van den Noort, J. C. Effect of walking with a modified gait on activation patterns of the knee spanning muscles in people with medial knee osteoarthritis. Knee27, 198-206, doi:10.1016/j.knee.2019.10.006 (2020).

32. Cui, W. L. et al. Effects of Toe-Out and Toe-In Gaits on Lower-Extremity Kinematics, Dynamics, and Electromyography. Applied Sciences-Base/ 9, doi:10.3390/app9235245 (2019).

33. Bennour, S., Ulrich, B., Legrand, T., Jolles, B. \& Favre, J. Effects of foot progression angle on knee biomechanics during gait modification. Comput Methods Biomech Biomed Engin 20, 17-18, doi:10.1080/10255842.2017.1382839 (2017).

34. Uhlrich, S. D., Silder, A., Beaupre, G. S., Shull, P. B. \& Delp, S. L. Subject-specific toe-in or toe-out gait modifications reduce the larger knee adduction moment peak more than a non-personalized approach. J Biomech 66, 103-110, doi:10.1016/j.jbiomech.2017.11.003 (2018).

35. Zeighami, A., Dumas, R. \& Aissaoui, R. Knee loading in OA subjects is correlated to flexion and adduction moments and to contact point locations. Sci Rep 11, 8594, doi:10.1038/s41598-021-87978-2 (2021).

36. Hoch, M. C. \& Weinhandl, J. T. Effect of valgus knee alignment on gait biomechanics in healthy women. J Electromyogr Kinesio/35, 17-23, doi:10.1016/j.jelekin.2017.05.003 (2017).

37. Böhm, H., Stief, F., Sander, K., Hösl, M. \& Döderlein, L. Correction of static axial alignment in children with knee varus or valgus deformities through guided growth: Does it also correct dynamic frontal plane moments during walking? Gait Posture 42, 394397, doi:10.1016/j.gaitpost.2015.06.186 (2015).

38. Trinler, U., Schwameder, H., Baker, R. \& Alexander, N. Muscle force estimation in clinical gait analysis using AnyBody and OpenSim. J Biomech 86, 55-63, doi:10.1016/j.jbiomech.2019.01.045 (2019).

39. Kainz, H. et al. Reliability of four models for clinical gait analysis. Gait Posture 54, 325-331, doi:10.1016/j.gaitpost.2017.04.001 (2017).

40. Moreland, J. R., Bassett, L. W. \& Hanker, G. J. Radiographic analysis of the axial alignment of the lower extremity. J Bone Joint Surg Am 69, 745-749, doi:10.2106/00004623-198769050-00016 (1987).

41. Paley, D. in Principles of Deformity Correction Ch. 1, 1-18 (Springer-Verlag Berlin Heidelberg, 2002).

42. Ruchholtz, S. \& Wirtz, D. C. in Orthopädie und Unfallchirurgie essentials Essentials - Intensivkurs zur Weiterbildung (eds Steffen Ruchholtz \& Dieter Christian Wirtz) (Georg Thieme Verlag, 2013).

43. Stief, F., Bohm, H., Michel, K., Schwirtz, A. \& Doderlein, L. Reliability and accuracy in three-dimensional gait analysis: a comparison of two lower body protocols. J App/ Biomech 29, 105-111, doi:10.1123/jab.29.1.105 (2013).

44. Kadaba, M. P., Ramakrishnan, H. K. \& Wootten, M. E. Measurement of lower extremity kinematics during level walking. $J$ Orthop Res 8, 383-392, doi:10.1002/jor.1100080310 (1990).

Page $12 / 14$ 
45. Stief, F. in Handbook of Human Motion Ch. Chapter 26, 509-526 (Springer International Publishing, 2018).

46. Hunt, M. A., Fowler, P. J., Birmingham, T. B., Jenkyn, T. R. \& Giffin, J. R. Foot rotational effects on radiographic measures of lower limb alignment. Can J Surg 49, 401-406 (2006).

47. Seth, A. et al. OpenSim: Simulating musculoskeletal dynamics and neuromuscular control to study human and animal movement. PLoS Comput Biol 14, e1006223, doi:10.1371/journal.pcbi.1006223 (2018).

48. Mantoan, A. et al. MOtoNMS: A MATLAB toolbox to process motion data for neuromusculoskeletal modeling and simulation. Source Code Biol Med 10, 12, doi:10.1186/s13029-015-0044-4 (2015).

49. Uhlrich, S. D., Jackson, R. W., Seth, A., Kolesar, J. A. \& Delp, S. L. Muscle coordination retraining inspired by musculoskeletal simulations: a study on reducing knee loading. bioRxiv, doi:10.1101/2020.12.30.424841 (2021).

50. Seagers, K. et al. Changes in foot progression angle during gait reduce the knee adduction moment and do not increase hip moments in individuals with knee osteoarthritis. medRxiv, 2022.2001.2010.22268858, doi:10.1101/2022.01.10.22268858 (2022).

51. Millard, M., Uchida, T., Seth, A. \& Delp, S. L. Flexing computational muscle: modeling and simulation of musculotendon dynamics. J Biomech Eng 135, 021005, doi:10.1115/1.4023390 (2013).

52. Silder, A., Whittington, B., Heiderscheit, B. \& Thelen, D. G. Identification of passive elastic joint moment-angle relationships in the lower extremity. J Biomech 40, 2628-2635, doi:10.1016/j.jbiomech.2006.12.017 (2007).

53. Stief, F. et al. Non-invasive determination of frontal plane lower limb alignment using motion capture technique - An alternative for full-length radiographs in young patients treated by a temporary hemiepiphysiodesis? Gait Posture 79, 26-32, doi:10.1016/j.gaitpost.2020.04.011 (2020).

54. Fritz, C. O., Morris, P. E. \& Richler, J. J. Effect Size Estimates: Current Use, Calculations, and Interpretation (vol 141, pg 2, 2011). Journal of Experimental Psychology-General 141, 30-30, doi:10.1037/a0026092 (2012).

55. Cohen, J. Statistical Power Analysis for the Behavioral Sciences. 2nd edn, (Lawrence Erlbaum Associates, 2013).

56. Pataky, T. C., Robinson, M. A. \& Vanrenterghem, J. Vector field statistical analysis of kinematic and force trajectories. J Biomech 46, 2394-2401, doi:10.1016/j.jbiomech.2013.07.031 (2013).

57. Wesseling, M., Meyer, C., Corten, K., Desloovere, K. \& Jonkers, I. Longitudinal joint loading in patients before and up to one year after unilateral total hip arthroplasty. Gait Posture 61, 117-124, doi:10.1016/j.gaitpost.2018.01.002 (2018).

\section{Figures}



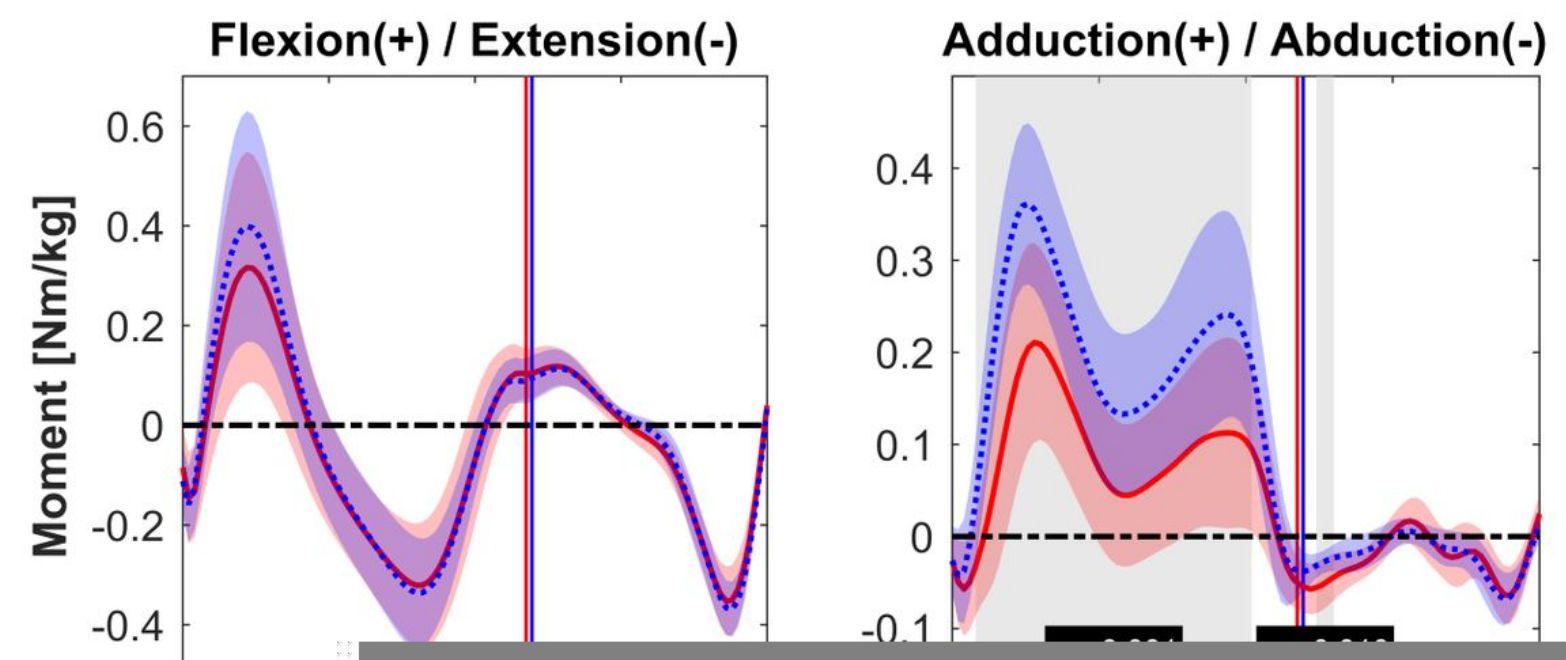

\section{Figure 1}

The mean (line) and standard deviation (shaded) of the external knee flexion and adduction moment and the medial and lateral knee contact force of the patients with valgus malalignment (red, solid) and the typically developed healthy controls (blue, dashed) are displayed. Vertical lines mark the end of the stance phase. Joint moments were normalized by body mass (unit: $\mathrm{Nm} / \mathrm{kg}$ ) and the joint contact forces by bodyweight (unit: $\mathrm{N} /(\mathrm{kg} \times \mathrm{ms}-2)$ ). Significant different phases $(\mathrm{p}<0.05)$ during the gait cycle (normalized to $100 \%$ ) calculated with a statistical parametric mapping two-sample t-test are highlighted with gray areas and are described with the associated p-value (black boxes).

\section{Supplementary Files}

This is a list of supplementary files associated with this preprint. Click to download.

- Supplementaryinformation.pdf 